\title{
Experimental Investigation of a Probe-Induced Localized Electron Temperature Elevation Near Electron-Cyclotron Resonance
}

\author{
RICHARD R. METT, SHU WAI LAM, AND JOHN E. SCHARER
}

\begin{abstract}
A spacially localized electron temperature elevation is observed near the electron-cyclotron resonance region. Detailed properties of this phenomenon are examined. The dependence of the temperature elevation upon launched wave amplitude and frequency relative to the local electron-cyclotron frequency, plasma density, neutral pressure, microwave cavity plasma source tuning, and probe type is discussed. These results are combined with detailed wave electric and magnetic field, emissive probe, and spectral measurements and the use of different Langmuir probe types and orientations to characterize the temperature elevation, background plasma, and wave properties. It is found that the magnetic field and wave absorption near the electroncyclotron resonance, probe type, and probe tip input impedance as seen from the plasma have important influences on the temperature elevation process. Two-probe correlation measurements are used to show that the elevation process exists in the radial neighborhood $(<1$ $\mathrm{cm}$ ) of the probe tip and along a flux tube parallel to the magnetic field. This process can be present in all Langmuir probe-diagnosed ECRH experiments and can be suppressed by appropriate probe design. Measurements suggest that the electron temperature elevation is due to the interaction of sufficiently strong wave electric fields near electron-cyclotron resonance with an appropriate probe tip input impedance as seen by the fields.
\end{abstract}

\section{INTRODUCTION}

$\mathrm{W}$ E present detailed measurements and interpretations of a spacially localized electron temperature elevation observed under a wide range of plasma source conditions, together with a range of launched frequencies for a right-hand circularly polarized (RHCP) wave launched below the electron-cyclotron frequency in an inhomogeneous magnetic field. Preliminary work on this subject has been carried out by Rice and Scharer [1]. This work determined the existence of the elevation and radial spacial uniformity and circular polarization of the wave. A model based on a single particle mechanism with electron-neutral collisions causing a change in the pitch angle of the resonantly heated electrons was proposed to describe the electron temperature elevation and relate the apparent axially localized electron temperature to the background electron temperature distribution.

Manuscript received January 18, 1989; revised June 27, 1989. This work was supported by the National Science Foundation under Grant ECS8514978.

The authors are with the Department of Electrical and Computer Engineering, University of Wisconsin. 1415 Johnson Drive, Madison, WI 53706.

IEEE Log Number 8930587
Earlier work by McVey and Scharer [2], [3] examined the theoretical hot-plasma, collisionless, spatial damping rate for an RHCP electromagnetic wave, and experimentally verified the dispersion and electron-cyclotron damping rates for a low power wave $(50 \mathrm{~mW})$ near and far from resonance in a weak magnetic field gradient that satisfied WKB criteria. Pulsed diamagnetic loop measurements by Owens and Scharer [1] at higher power levels proved that the wave damping corresponds to an increase in plasma diamagnetism in the region of absorption. Later work by Scharer and Mitzlaff [5] experimentally examined the lower sideband and low-frequency parametric instabilities caused by launching waves at power levels of 10 to $15 \mathrm{~W}$. More recent work on another experiment by Booske et al. [6] examined the electron temperature anisotropy and whistler instability spectra that can be created by sufficiently high power pulsed heating in a magnetic mirror.

Our measurements have focused on the dependence of the temperature elevation on wave amplitude and frequency, plasma source input power and matching conditions, and neutral pressure. Detailed observations have been made of the received wave amplitude profile, sideband spectrum, cold electron-cyclotron resonance position, and plasma parameters in the vicinity of the elevation. Measurements are performed in the steady-state using Langmuir, emissive, and small antenna probes. We have carried out measurements with different Langmuir probe types, and two-probe correlation measurements are used to show that the temperature elevation process exists in the radial neighborhood of the probe tip and along a flux tube parallel to the magnetic field.

\section{EXPERIMENTAL Description}

\section{A. Experimental Configuration}

The experimental layout is shown in Fig. 1. The measurements are carried out inside a glass tube $15.2 \mathrm{~cm}$ in inner diameter, $200 \mathrm{~cm}$ in length, and wrapped with copper screen to provide shielding and a well-defined RF boundary condition. A diffusion pump provides a base pressure of $1 \times 10^{-6}$ torr. Eight water-cooled magnetic field coils are aligned to produce the on-axis axial magnetic field profile shown in Fig. 1. The associated axial magnetic field gradient is shown with a dashed line. The 


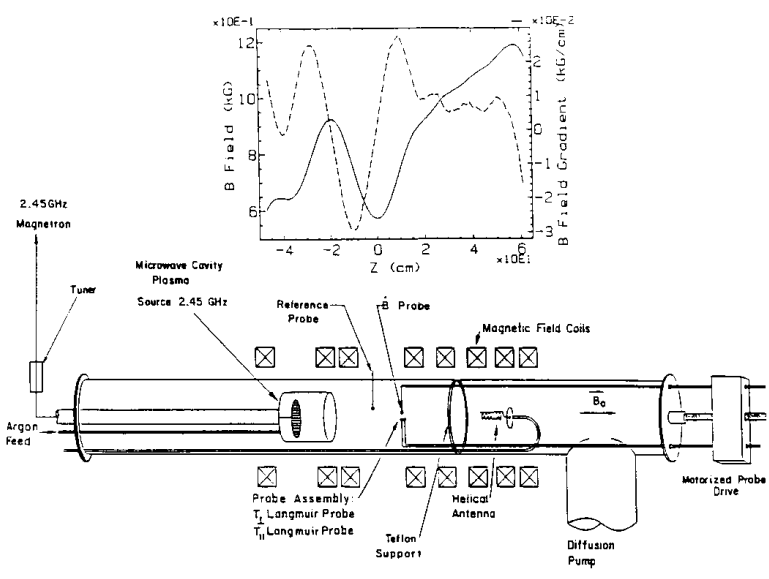

Fig. 1. Experiment configuration with superimposed dc magnetic field (solid line) and gradient (dashed line). The total magnet current is $I=$ $280.0 \mathrm{~A}$. Note that the magnetic field can be obtained for all other cases by utilizing these curves.

location of the magnetic field minimum, inferred from a least-squares parabolic fit to the data for $-3<z<3 \mathrm{~cm}$, is defined as $z=0$. The central mirror extends from -20 to $20 \mathrm{~cm}$, with a mirror ratio of 1.6 .

Most experimental wave data is taken in the region $3<z<18 \mathrm{~cm}$. The microwave cavity plasma source, positioned in the region $-40<z<-30 \mathrm{~cm}$, is driven by a $2.45-\mathrm{GHz}$ magnetron at power levels from 70 to 200 $\mathrm{W}$. The plasma is created by electron-cyclotron resonance heating (ECRH) in the cavity, with argon at pressures ranging from $8 \times 10^{-5}$ to $10^{-3}$ torr. Electron-cyclotron waves excited by the source are evanescent for $z>-13$ $\mathrm{cm}$. Residual-source RF levels in the region where most data is taken are typically below $-40 \mathrm{dBm}$ as measured with the shielded RF loop antenna probe. A moveableprobe waveguide tuner between the isolator-protected magnetron output and plasma source allows variation in the matching between these devices. The plasma parameters can be varied by adjusting the magnetron power output, the matching, and the magnetic field. The background electron temperature is $3<T_{e}<4 \mathrm{eV}$, while the ion temperature has been estimated to be $\leq 0.1 \mathrm{eV}$ by the vacuum monochrometer method. The background plasma density is in the range $1<n<9 \times 10^{10} \mathrm{~cm}^{-3}$ as determined by Langmuir probe measurements. A helical launch antenna in the region $35<z<60 \mathrm{~cm}$, where $f / f_{c e}<$ 0.8 is driven in the frequency range $1.90<f<2.30 \mathrm{GHz}$ by a traveling-wave tube with a maximum power output of $20 \mathrm{~W}$. Earlier experiments [1], [2] verified that this type of antenna launches an RHCP wave with respect to the magnetic field when $f_{p}>f$.

\section{B. Data Acquisition and Analysis}

Data acquisition and analysis are performed with an IBM PC/AT and Metrabyte Dash-16 data acquisition board. A signal conditioning unit converts the signals from the Langmuir and emissive probes and laboratory equip- ment into the \pm 5 -V range, utilizing all 12 bits of resolution of the Dash-16. This unit also sweeps the Langmuir and emissive probes and allows the AT to sweep the spectrum analyzer and boxcar integrator, as shown in Fig. 2. Signal conditioning is performed by AD524/624 instrumentation amplifiers. Data is recorded by custom TurboPascal 4.0 programs and archived for subsequent analysis.

The axial position is measured by means of a geardriven precision ( 0.025 percent linearity error) 10 -turn potentiometer. The total axial position error is $\pm 0.1 \mathrm{~cm}$. The axial magnetic field profile at various radii has been measured to \pm 0.37 percent, $\pm 1.0 \mathrm{G}$ traceable to the NBS with an F. W. Bell model 810 Hall effect field monitor. The magnetic field data is normalized to the magnet current measured by current shunts during each experiment and is used with the wave frequency (accurate to within \pm 0.05 percent) to compute the cold electron-cyclotron resonance axial position. Typical resonance positions are accurate to within $\pm 0.3 \mathrm{~cm}$.

The wave magnetic field is measured by means of an RF loop antenna probe consisting of a $0.75-\mathrm{cm}$ diam electrostatically shielded loop oriented perpendicularly to the dc magnetic field. It is constructed from a single turn of $8.6 \times 10^{-2} \mathrm{~cm}$ diam high-temperature semi-rigid coaxial cable. Its output drives a directional coupler, which in turn drives a spectrum analyzer and an interferometer, as shown in Fig. 2.

An indication of the wave transverse electric field is obtained by connecting a Langmuir probe in series with a microwave feed-through capacitor to the same apparatus used to measure the wave magnetic field. The Langmuir probe stalks are $6 \mathrm{~cm}$ in length, aligned perpendicularly to the magnetic field and shielded from the plasma with ceramic tubing. The signal is carried from the machine through a $0.12-\mathrm{cm}$ diam semi-rigid coaxial cable. In this configuration the probe functions as a monopole antenna.

Plasma density and electron temperature are measured by means of Langmuir probes consisting of $0.24-\mathrm{cm}$ diam tantalum discs $1.3 \times 10^{-2} \mathrm{~cm}$ in thickness. We find that these give an estimate of the electron thermal anisotropy by orienting their surface normals parallel or perpendicular to the dc magnetic field. Phase-sensitive measurements of wavelength far from resonance at a known magnetic field when inserted into the cold plasma whistler dispersion relation for $f_{p}>f$ yield an inferred plasma density to within 7 percent of the measured perpendicular probe plasma density.

Various types of disc Langmuir probe stalks were used, which responded differently to the RF wave fields. These are shown in Fig. 3. The unshielded Langmuir probe (Fig. 3(b)) stalk is of a standard design, with a straight single conductor to the tip insulated from the plasma by ceramic tubing but exposed to the wave fields, which can penetrate the dielectric. (Note that all stalks begin at a connector housing at a $7-\mathrm{cm}$ radius just outside the plasma. Signals are carried out of the machine via semi-rigid coaxial cable.) The coaxially shielded Langmuir probe stalk (Fig. 


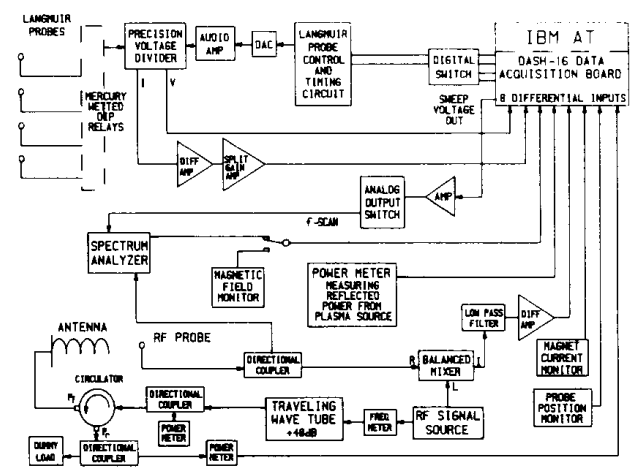

Fig. 2. Data acquisition system block diagram.
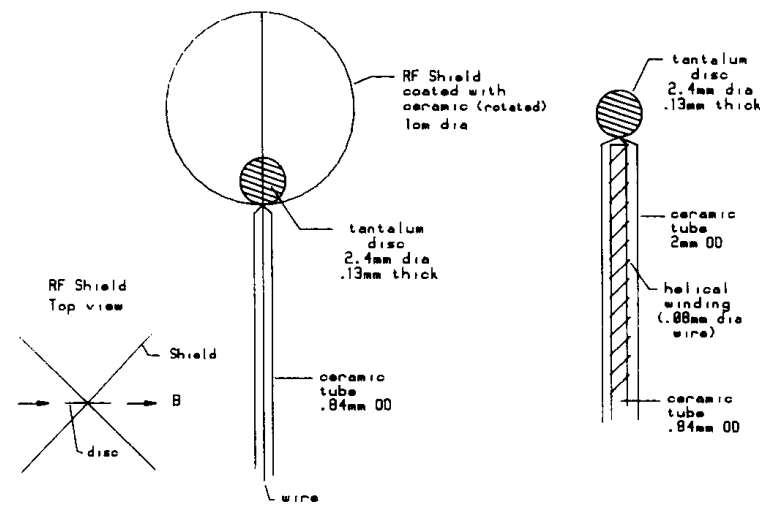

(a)
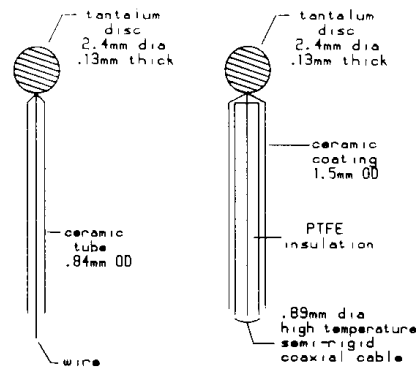

(b)

Fig. 3. Various types of disc Langmuir probes. (a) RF shielded and helical probes. (b) Unshielded Langmuir and coaxially shielded probes.

3(b)) consists of high-temperature semi-rigid coaxial cable whose outer conductor is at ground potential and shielded from the plasma by ceramic tubing. The exposed Teflon insulation at the tip is covered with a ceramic coating. This probe provides a far more localized wave field measurement than the unshielded Langmuir probe at the wave frequency when it is properly terminated with $50 \Omega$. The weakly RF-shielded disc Langmuir probe (Fig. 3(a)) is a modification of the unshielded Langmuir probe, where two 1-cm diam loops of gold-plated wire encircle the tantalum disc and are connected to the disc feed wire just below its base. The gold-plated wires are at right angles with respect to one another to partially shield the tip from the RF fields. These are coated with ceramic insulation and are aligned so that the magnetic flux lines intersecting the probe tip are not obstructed. The helical probe (Fig. 3(a)) consists of a tantulum disc connected to a 17-turn/ $\mathrm{cm}$ helix of fine copper wire wound on a ceramic core and shielded from the plasma by ceramic tubing. The helix has an inductive impedance of about $500 \Omega / \mathrm{cm}$ at 2.00 $\mathrm{GHz}$.

The plasma potential is measured by means of an emissive probe consisting of a $2.5 \times 10^{-3} \mathrm{~cm}$ diam tungsten filament $1 \mathrm{~cm}$ in length and heated by a dc current of about $140 \mathrm{~mA}$. The filament supply is floating, which allows the probe to be swept with the apparatus used to sweep the Langmuir probes. Two identical precision resistors across the filament give the average dc voltage on the filament. The inflection point method [7] under conditions of low electron emission is used to determine the plasma potential from its characteristic curve. This method is less perturbing than others and is accurate in measuring small changes in the plasma potential.

\section{Experimental Results}

\section{A. Temperature Elevation for $f_{p}>f$}

Shown in Fig. 4 are representative data for the electron bulk temperature and plasma density measured by a perpendicularly oriented unshielded Langmuir probe (shown in unfilled diamonds) and the relative power and wave phase received by the RF loop-antenna probe. The relative power is the peak power level on a spectrum sweep in the vicinity of the wave frequency with a bandwidth of $300 \mathrm{kHz}$. These data correspond to two higher density ( $n$ $=8 \times 10^{10} \mathrm{~cm}^{-3}$ ) short wavelength cases where the wave has an electromagnetic whistler character with $f_{p} / f=1.2$. A reference perpendicular, unshielded Langmuir probe (shown as filled diamonds) is used to monitor the background plasma characteristics at a fixed position near the magnetic minimum to ensure that the plasma characteristics do not change as the other probes are moved through the elevation region. The plasma characteristics from this probe are plotted versus the axial position of the movable probe. The cold electron-cyclotron resonance position is shown as a solid vertical line.

In Fig. 4 the transverse temperature increases by about a factor of two above the background level of $3.6 \mathrm{eV}$. The net launched wave power level is $13.4 \mathrm{~W}$, with $1.6 \mathrm{~W}$ reflected from the helical wave launcher. The probe plasma density varies by less than 10 percent from one side of the temperature elevation location, where the strong wave amplitude is present, to the other side beyond cyclotron resonance, where the wave power is weak $(-40$ $\mathrm{dB}$ ). This indicates that the Langmuir probe measurements are not significantly perturbed by the wave. Typical wavelengths near resonance are $2 \mathrm{~cm}$. The RF loop-antenna probe indicates that the received spacial power flux first decreases as the temperature begins to increase, then flattens and increases slightly as the electron temperature 


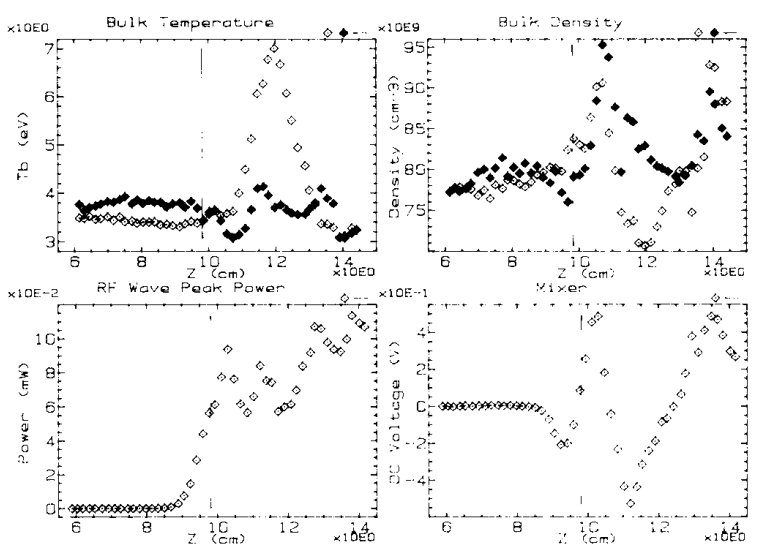

Fig. 4. Typical large temperature elevation for $f_{p}>f$ measurements performed with perpendicularly fixed (filled diamond) and moveable (unfilled diamond) Langmiur probes; $f=2.122 \mathrm{GHz} . l=284.8 \mathrm{~A}$.

decreases again, therefore quickly dropping to a very low level after cyclotron resonance is passed. The RF loopantenna probe power typically decreases by 35 to $50 \mathrm{~dB}$ beyond cyclotron resonance. This case is typical of higher density temperature elevations with a significant rise in temperature.

It should be noted that the size of the temperature elevation is dependent upon the tuning of the microwave cavity plasma source. After retuning the cavity so that the reflected power from the source is decreased with the forward power constant, the plasma density typically increases by 15 percent, and the typical peak of the temperature elevation decreases significantly from 6.5 down to $4.5 \mathrm{eV}$. The temperature elevation is smaller at higher densities because the available wave power per particle is lower.

\section{B. Langmuir Probe Characteristics}

Typical (unshielded) Langmuir probe traces are shown in Fig. 5. In the left column is the linear $I$ versus $V$ characteristic; in the middle is the analyzed $\ln \left(I-I_{i s}\right)$ versus $V$ curve (log trace); and on the right is the curvature of the log trace from which the knee is found. The top row of traces are from a parallel probe in a region away from a temperature elevation with a strong RF wave present. The knee is prominent. The middle row of traces are for a perpendicular probe under similar conditions. The knee is not as prominent as for the parallel probe due to the effect of the strong magnetic field. The bottom row of traces are for a perpendicular probe in the temperature elevation region. The nearly straight-line log trace over three orders of magnitude in current indicates a nearly Maxwellian distribution.

Varying the neutral pressure has been observed to have a particular effect on the perpendicular Langmuir probe characteristic curve in both the higher and lower density regimes. In the temperature elevation region, as the pressure is varied from 1.5 to $9 \times 10^{-4}$ torr, both the plasma potential and floating potential decrease by about 4 and 3
$\mathrm{V}$, respectively. At higher neutral pressures the probe log trace shows a gradual increase in slope from $V_{p}$ down to -10 to $-5 \mathrm{~V}$. This implies that electrons of higher kinetic energy have a lower temperature than those of lower kinetic energy. Neutrals produce a cool electron population in the velocity distribution function. This effect is slight at $1.5 \times 10^{-4}$ torr, while it is quite prominent at $9 \times 10^{-4}$ torr. Therefore neutrals affect the perpendicular electron velocity distribution under all operating conditions. This effect is observed in the parallel Langmuir probe characteristic only when neutral pressures exceed $8 \times 10^{-4}$ torr. We interpret these results to be an observation of a Druyvesteyn-like [8] electron distribution in perpendicular velocity. A purely elastic collision cross section that is independent of particle energy gives rise to the Druyvesteyn distribution, whereas the Coulomb collision cross section, which is porportional to the inverse square of the electron energy, gives rise to the Maxwell distribution. As a first approximation, the elastic electron-argon collision cross section is relatively independent of the electron energy from 5 to $20 \mathrm{eV}$ [9].

Extensive measurements were performed with an emissive probe. These results showed that no electrostatic potential structure exists to axially confine electrons in the temperature elevation region. We typically saw that the plasma potential varied by 0.5 to $1 \mathrm{~V}$ through the temperature elevation region, but it did not show a corresponding axial structure for an observed temperature elevation structure under different operating conditions, even though the results were very repeatable under the same operating conditions. We found that the plasma potential was proportional to the net coupled wave power, which varied as the probes were moved axially through the temperature elevation region. This is strong evidence for an interaction between the wave, probe, and plasma which produces the temperature elevation. The plasma potential variations agreed with those measured by means of the Langmuir probes in all cases.

\section{Temperature Elevation for $f_{p}<f$}

When the plasma source is retuned and the plasma density is reduced so that $f_{p}=0.8 f$, typical data for an unshielded Langmuir probe with an RF loop-antenna probe appear as shown in Fig. 6. At the lower density the net coupled power is reduced to $8 \mathrm{~W}$, with $7 \mathrm{~W}$ reflected due to the mismatch between the launcher helix periodicity and the lower density plasma requiring a longer wavelength. We also used the Langmuir probe tip as a wave power receiver by taking part of the signal and sending it via a directional coupler to a spectrum analyzer tuned to the input frequency. The wave power received by the probe tip as it is moved is highly peaked and correlated with the temperature elevation process in this case. The mixer trace shows a long parallel wavelength character with substantial phase and amplitude variations near the resonance zone. In the regime where $f_{p}<f$, the phase information detected by either the electrostatic probe tip or the loop antenna probe does not show a characteristic 

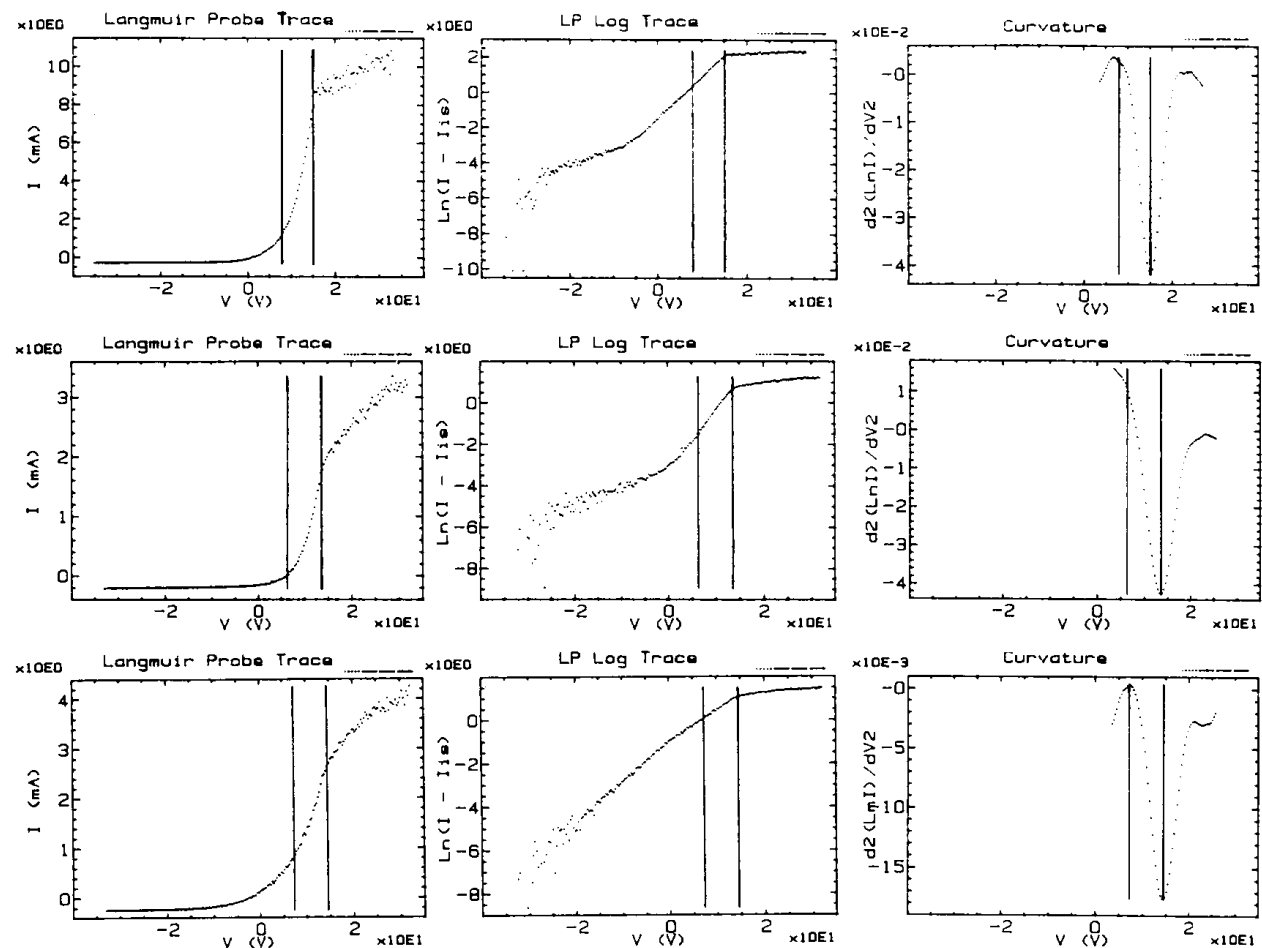

Fig. 5. Typical Langmuir probe traces for $f=2.122 \mathrm{GHz}$ and $I=284.8 \mathrm{~A}$ : Left-linear $I$ versus $V$ characteristic; middle$\ln \left(I-I_{i s}\right)$ versus $V$ trace; right-curvature versus $V$. Top-parallel probe; middle-perpendicular probe away from temperature elevation; bottom-perpendicular probe in the temperature elevation.
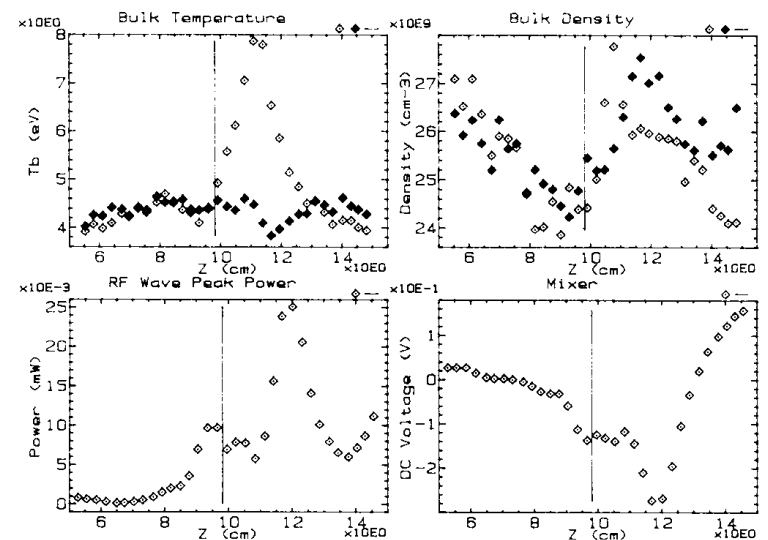

Fig. 6. Typical temperature elevation for $f_{p}<f$ at $f=2.122 \mathrm{GHz}$ and $I$ $=283.5 \mathrm{~A}$.

longitudinal phase variation that one would expect for an electron-cyclotron wave. This is because the helical wave launcher, which has a pitch periodicity of about $1 \mathrm{~cm}$, does not correspond to the $7-\mathrm{cm}$ wavelength near the launcher that one would expect for the cyclotron wave mode. The net coupled wave power is reduced to $8 \mathrm{~W}$ in this regime, with less well-defined detected phase information in the wave propagation region. We attribute this to the helical radiator launching waves at larger angles, and with more electrostatic mode content than in the $f_{p}>$ $f$ case. Yet we notice that the temperature elevation process is still present, with a similar spacial excitation profile for $f_{p}<f$ when the neutral pressure is varied over about an order of magnitude. We therefore conclude that the background neutral density level does not play an important role in the temperature elevation process.

\section{Influence of Wave Frequency and Wave Power}

Fig. 7 shows the axial temperature elevation profile detected by a perpendicular, coaxially shielded Langmuir probe as a function of the wave frequency from 1.89 to $2.32 \mathrm{GHz}$ for fixed plasma-source operating conditions when $f_{p}>f$, with a net coupled power of $13 \mathrm{~W}$. These axial profiles are presented with respect to the corresponding cold electron-cyclotron resonance positions in Table I.

It is noted that typically the leading edge of the elevation occurs near $f / f_{c e}=0.90$, with a peak near $f / f_{c e}=$ 0.95 and the trailing edge near $f / f_{c e}=0.99$. The width of the elevation process is greater than the theoretical collisionless cyclotron damping width [2] for a 4-eV Maxwellian, which would yield appreciable damping at $f / f_{c e}$ $=0.95$ and above for these plasma parameters. We anticipate that the larger shift from resonance of the electron-cyclotron heating process comes from a drifting Maxwellian component from the source. This component could have parallel energies large enough to cause them 


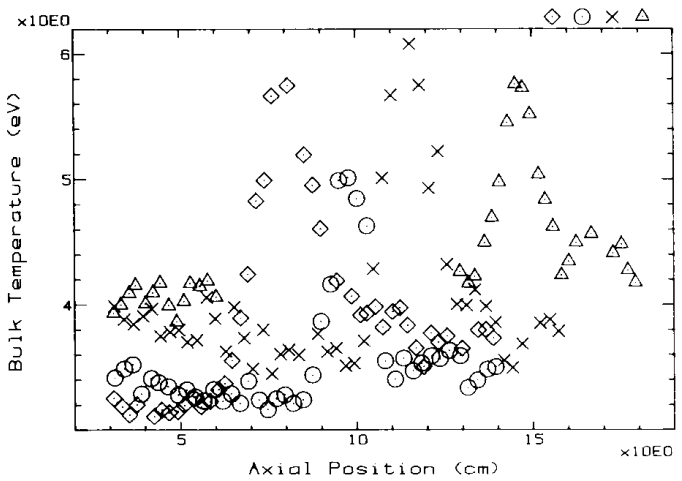

Fig. 7. Influence of wave frequency on the temperature elevation: $(0)=$ $1.890 \mathrm{GHz} ;(+)=2.100 \mathrm{GHz} ;(\times)=2.180 \mathrm{GHz} ; \triangle)=2.320 \mathrm{GHz}$.

TABLE I

Axial Profilf of $T$. Elevation

\begin{tabular}{llll}
\hline \hline $\begin{array}{l}\text { frequency } \\
(\mathrm{GHz})\end{array}$ & $\begin{array}{l}\mathrm{f} / \mathrm{f}_{\mathrm{ce}} \\
(\text { trail })\end{array}$ & $\begin{array}{l}\mathrm{f} / \mathrm{f}_{\mathrm{ce}} \\
(\text { peak })\end{array}$ & $\begin{array}{l}\mathrm{f} / \mathrm{f}_{\mathrm{ce}} \\
(\text { lead })\end{array}$ \\
\hline 1.89 & 0.98 & 0.93 & 0.87 \\
2.10 & 1.00 & 0.96 & 0.91 \\
2.18 & 1.00 & 0.95 & 0.90 \\
2.32 & 0.98 & 0.95 & 0.92 \\
\hline
\end{tabular}

to be heated far from resonance. Fig. 8 illustrates a plot of the power in $\mathrm{dBm}$ received by the single coaxially shielded probe, with a $300-\mathrm{kHz}$ bandwidth about the launched frequency as a function of the net coupled wave power in $\mathrm{dB}$. The frequency is $2.04 \mathrm{GHz}$, and the square points denote the case where $f_{p}<f$ and the circles denote the case where $f_{p}>f$. Note that both cases have a linear dependence. For $f_{p}>f$ the temperature elevation was not observable at net power levels below $3 \mathrm{~W}$. As the wave power was increased from 3 to $15 \mathrm{~W}$, the temperature peak monotonically increased from 0.5 to $4 \mathrm{eV}$ above the background. For the case of $f_{p}<f$, results were very similar to those for $f_{p}>f$ except that the temperature elevation was observable at a power level of $0.5 \mathrm{~W}$ due to the lower plasma density.

It is interesting to note that the case where $f_{p}>f$ has a lower sideband for which the received spectral shape as a function of probe position is shown in Fig. 9. (Such spectra are observed with both the RF loop antenna probe and Langmuir probes.) Note that a broad lower sideband is present, which decays along with the launched signal as resonance is approached. In the case of a launched wave for $f_{p}<f$, no sidebands are observed about the launched frequency.

\section{E. Single-Probe Measurements}

Shown in Fig. 10 are the bulk temperature, density, received power, and relative wave phase, all measured with a single coaxially shielded probe in a higher density plasma $\left(f_{p}=1.2 \mathrm{f}\right)$ at a wave frequency of $1.94 \mathrm{GHz}$. Only one probe is present in the system to eliminate pos-

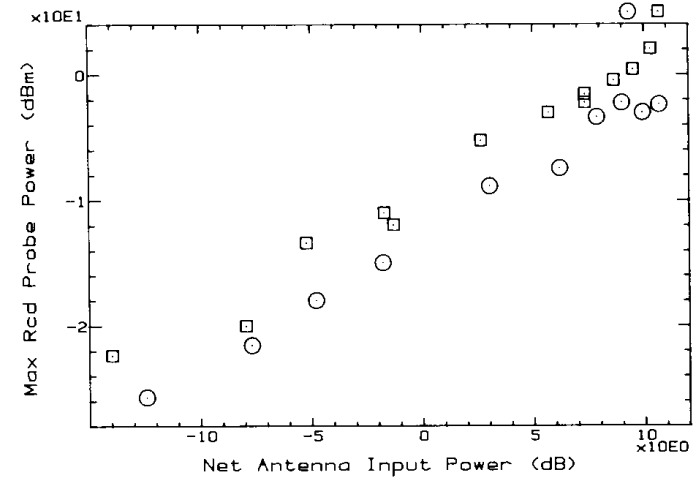

Fig. 8. Power received by a single coaxially shielded Langmuir probe as a function of the net launched wave power for $f_{p}>f$ and $f_{p}<f, f=$ 2. $120 \mathrm{GHz}$.

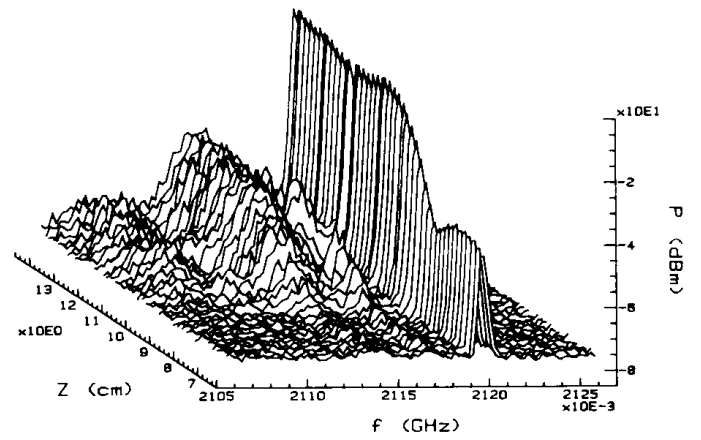

Fig. 9. Wave power spectra as a function of the axial position at $f=2.120$ $\mathrm{GHz}$ and $I=283.5 \mathrm{~A}$.
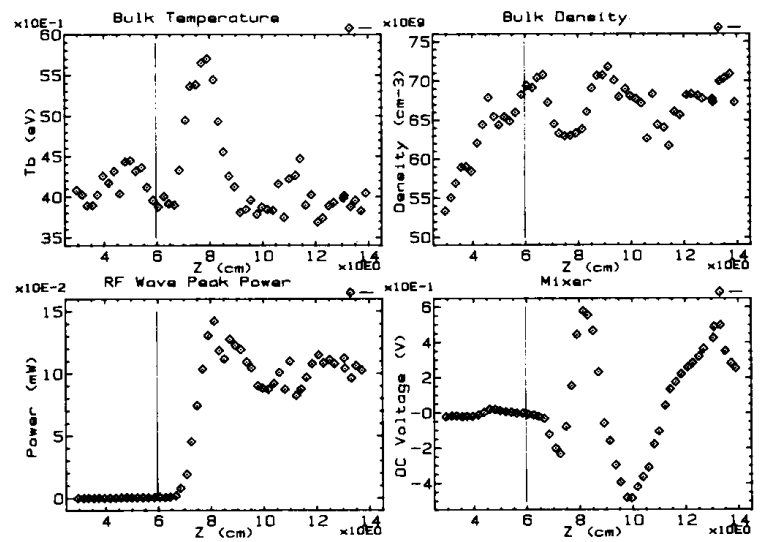

Fig. 10. Electron temperature, density, RF power, and relative phase measured from a single coaxially shielded Langmuir probe on-axis: $f_{p}>$ $f$ for $f=1.940 \mathrm{GHz}$ and $I=300.4 \mathrm{~A}$.

sible probe interactions. The axial position of the cold plasma cyclotron resonance is denoted by a vertical line near the 6-cm mark. The temperature elevation exists over a $2-\mathrm{cm}$ axial range of probe positions with a corresponding peak in the received wave power. The net launched 
wave power is $13 \mathrm{~W}$, with $2 \mathrm{~W}$ reflected, although the relected power varied by $1-2 \mathrm{~W}$ as the probe was moved through the elevation region. Such results are quite repeatable and occur over a wide range of frequencies.

Shown in Fig. 11 is a comparison of the temperature elevation measured by a single coaxially shielded Langmuir probe (unshielded tip) with that measured by a single helical probe (see Fig. 3) under similar operating conditions. Note that the temperature elevation is drastically reduced by the helical probe. This effect was observed in both the higher density and lower density cases. The partially RF-shielded Langmuir probe (see Fig. 3) reduced the temperature elevation by a lesser amount than the helical probe.

\section{F. Two-Probe Correlation Measurements}

In order to determine the axial and radial extent of the temperature elevation, we carried out two-probe correlation measurements. This must be done with some care since wave scattering and plasma shadow effects from one probe can affect the measurements by the other. The first measurement was to place a perpendicular temperaturesensitive reference Langmuir probe near the magnetic field minimum and $5-\mathrm{cm}$ radially off the axis of the movable probe to monitor the background column plasma characteristics at a fixed position to ensure that the plasma characteristics do not change as the other probe is moved through the location of the elevation region. We found that the reference probe temperature did not vary appreciably $( \pm 0.3 \mathrm{eV}$, which is the usual range of variation when the launched wave is not present) when the perpendicular probe measured a $1.4-\mathrm{eV}$ rise from the background level for $f_{p}>f$. Next we used two probes with the disks oriented perpendicularly to the magnetic field, the stalks approaching each other from opposite plasma edge regions, and the axial and radial separation was changed for different runs. We used 3-5-cm axial separations which correspond to several wavelengths in the elevation zone, and the radial separation was varied from on-axis alignment to a $3-\mathrm{cm}$ radial separation.

Fig. 12 illustrates an interesting case in which the two coaxial probes were separated by $5 \mathrm{~cm}$ and aligned onaxis. Since the mirror magnetic field lines are diverging as one moves away from the wave launcher, it is never possible to exactly align the two probes, and this becomes more of a consideration as the probe axial separation is increased. One notes that the peaks in the bulk temperature for the two probes are separated by $5 \mathrm{~cm}$, and that a peak in probe 2 furthest from the launcher occurs when the wave amplitude received by probe 1 is peaked. The received wave power measured by probe 1 is seen to decrease to a negligible value at the position where probe 2 has a temperature peak furthest from the launcher. We carried-out aligned two-probe correlation measurements for both coaxial and straight-center conductor probes and found the correlation effects to be present for $f_{p}>f$ and $f_{p}<f$.

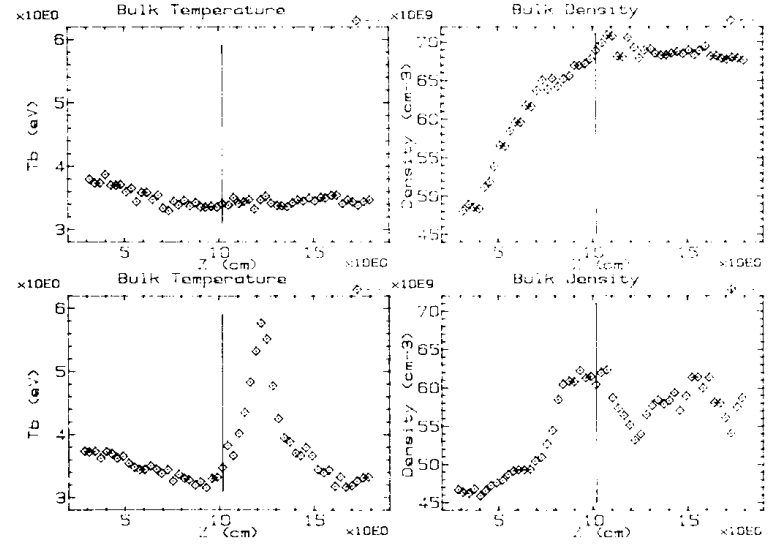

Fig. 11. Temperature elevations measured by a helical probe (above) and a coaxially shielded Langmuir probe (below).
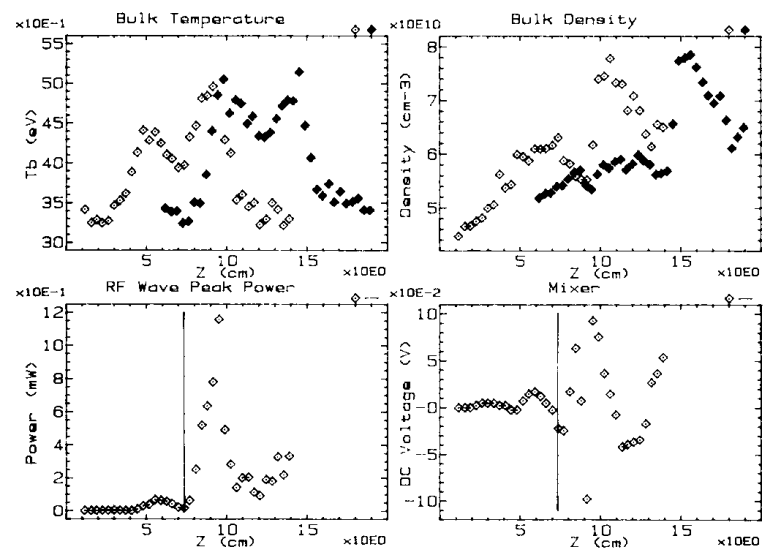

Fig. 12. Two on-axis probe correlation depicting temperature elevation extending along a flux tube.

Fig. 13 illustrates the temperature elevation for a case when $f_{p}>f$, where a $6.3-\mathrm{cm}$ axial separation and both on-axis and $1.5-\mathrm{cm}$ radial separations are made between a coaxially shielded Langmuir probe and a weakly RFshielded probe located closer to the wave launcher. The probes approach each other from opposite glass wall locations. In the top figure the on-axis correlation is good, indicating that the temperature elevation follows the flux line. In the bottom figure, where the coaxially shielded probe is $1.5-\mathrm{cm}$ off-axis, there is little correlation between the probe-measured temperatures, although scattering of the wave from one can affect the fields and elevation process of the other. We found that good correlations exist for the temperature elevation caused by one probe entering the temperature elevation zone near cyclotron resonance, influencing the plasma parameters as measured by the other for radial separations of $1 \mathrm{~cm}$ or less and axial separations of less than $6 \mathrm{~cm}$. This indicates that the heating and radial diffusion of the electrons near resonance occurred over a region that is not confined to the shadow of the probe tip. 


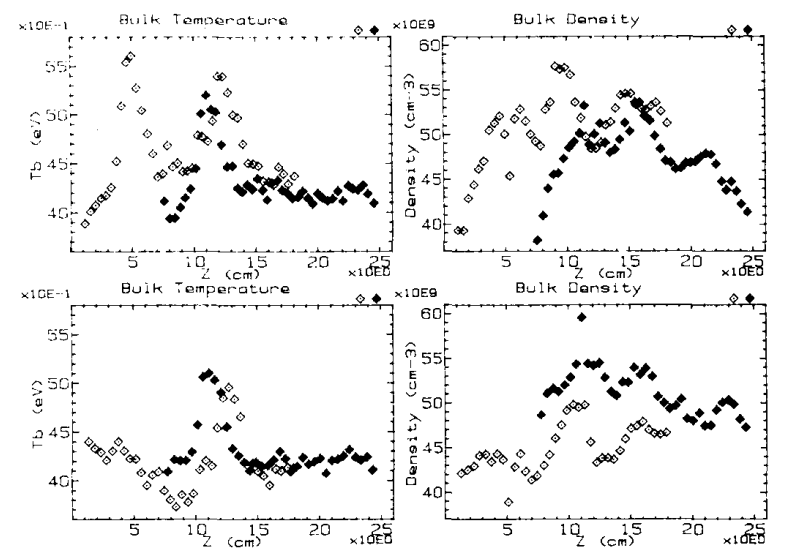

Fig. 13. Two-probe correlation measurements at $f=2.120 \mathrm{GHz}$ and $I=$ $280.0 \mathrm{~A}$.

\section{Analysis and Summary}

\section{A. Theoretical Wave Propagation Characteristics}

The wave propagation in our experiment involves wall radial boundary conditions which depend on the launched wave frequency, launcher polarization, and plasma parameters and yield different solutions dependent on these parameters. We begin from an unbounded description to analyze the wave character in different regimes. For $f_{p}>$ $f$ the wave is launched in the upper right quadrant of the Clemmow-Mullaly-Allis (CMA) diagram where $f / f_{c e}=$ 0.65 and $f_{p e} / f=1.2$. Here an RHCP electron-cyclotron wave propagates and has been measured to exist in the region between the helical wave launcher and the spacial position of electron-cyclotron resonance. It has a dumbbell-shaped phase velocity surface whose angle of propagation ranges from $\pi / 6$ with respect to the magnetic field near the launcher, down to zero as resonance is approached. In addition, a left/extraordinary $(l / x)$ mode can propagate possessing a spheroidal phase velocity surface with a magnitude greater than $c$. The wave launcher does not couple well to this long-wavelength mode.

When $f_{p}<f$ there is a left/ordinary $(l / o)$ mode with a long wavelength character and a phase velocity structure similar to the $l / x$ mode. A right/extraordinary $(r / x)$ mode remains axially resonant at the electron-cyclotron frequency. The $x$ part propagates past electron-cyclotron resonance at large angles with respect to the magnetic field to the upper hybrid resonance.

In the cold plasma limit the group velocity and direction of energy flow become perpendicular to the phase velocity near electron-cyclotron resonance for finite $k_{\text {perp }}$. This may lead one to conjecture that the wave power diverges out of the plasma column as the wave approaches resonance, accounting for the observed damping. However, ray-tracing studies by Batchelor et al. [10] that are valid near cyclotron resonance in parallel plane stratified plasmas have shown that for moderate $k_{\perp}$ and moderate plasma densities $f_{p} / f \sim 1$, damping is so strong that complete absorption can take place over a few perpendicular cen- timeters. This is supported by our observation that when the RF shielding was removed from the glass vacuum vessel, the plasma column radiated no microwave power except under very low density operating conditions. These ray-tracing studies have also shown that when finite temperature effects are included, waves propagating from higher density regions to lower density regions toward the upper hybrid resonance are reflected back from a perpendicular density gradient as a backward electrostatic wave [10]. This may occur in the lower density regime where up to 10 percent of the wave power propagates past cold electron-cyclotron resonance.

The WKB approximation conditions $k_{i} \ll k_{r}$ and $d k_{r} / d z \ll k^{2}[11]$ are not well satisfied in this experiment due to the relatively steep magnetic-field gradient. We compare the ratios of $k_{i}$ to $k_{r}$, experimentally determined from RF magnetic loop probe measurements near cold electron-cyclotron resonance (max exp), to those calculated from a code modeling the WKB hot plasma dispersion relation [2] for plasma parameters corresponding to the experiment (max thy). We find $\left(k_{i} / k_{r}\right)_{\max \exp }=0.25$ and $\left(\left(d k_{r} / d z\right) / k^{2}\right)_{\max e x p}=0.12$, while $\left(k_{i} / k_{r}\right)_{\max \text { thy }}=$ 1.0 and $\left(\left(d k_{r} / d z\right) / k^{2}\right)_{\max \text { thy }}=0.31$. Axial reflections of the wave are observed to be small since the wave power amplitude is relatively uniform far from the wave resonance and elevation region. A full wave treatment of wave propagation and absorption in a nearly plane stratified plasma, with a linear magnetic field gradient parallel to the magnetic field by Fruchtman et al. [12], with plasma parameters comparable to our experiment shows a notable deviation from a WKB treatment [2]. The results for parallel propagation show that the absorption is more complete and occurs over a shorter distance than that predicted by WKB theory. Unlike the WKB treatment, the wave power (Poynting vector magnitude) is shown to increase by 15 percent before dropping off as a function of axial position. Physically, this has been interpreted to be the result of particles with a positive parallel velocity having the correct phase relation to return energy to the wave, which had been absorbed from the wave in the region of strong interaction. Furthermore, the half-power point of wave absorption occurs at about a 3-percent higher magnetic field, farther from cold electron-cyclotron resonance than that predicted by WKB theory. Another interesting result from this work is that the full wave treatment predicts that half the power from the wave goes to particles with energies above $6 T_{e}$.

We have evaluated the collisionless cyclotron damping by using a WKB treatment [2] for a 4-eV Maxwellian with plasma and wave parameters corresponding to the higher density regime and found that appreciable damping should not occur until $f / f_{c e}=0.97$. As seen from Table I, this is larger than that typically observed at the peak $(0.94)$ of the temperature elevation and much larger than that observed for the leading edge (0.91) of the elevation. A drift velocity of the bulk electrons coming from the plasma source of $4 \mathrm{eV}$ can account for much of this upshift. We then obtain appreciable damping for $f / f_{c e}>0.935$. While 
this is close to the average position of the temperature elevation peak, it does not explain observed occurrences of large temperature elevation peaks as far from cold resonance as $f / f_{c e}=0.92$, with leading edges at $f / f_{c e}=$ 0.88 . Drifts as large as 25 to $40 \mathrm{eV}$ or higher densities than those measured by the Langmuir probes are necessary to account for these observations within this model, since the shift in resonance goes as the square root of the drift energy. A full wave treatment [12] offers an explanation, since the absorption is then downshifted in frequency $f / f_{c e}$ by another 3 percent, placing the absorption within 2 percent of the leading edge of the observed temperature elevation when an anisotropy of 1.3 and drift of $4 \mathrm{eV}$ from the source are also included.

\section{B. Collisional Effects}

Total elastic and inelastic electron-argon collision cross sections may be found in the literature [8], [9]. They do not become comparable to each other until the electron energies are $30 \mathrm{eV}$ or greater. We, therefore, do not expect that ionization will contribute to the elevation process unless some enhancing effect is present, such as strong RF fields at the probe tip. Using the elastic collision cross sections [8] in the equation

$$
\nu_{e n}=n_{o} \sigma_{e n}\left(k T_{e} / m_{e}\right)^{1 / 2}
$$

we compare in Table II the calculated electron-argon collision frequencies with the Coulomb collisions, calculated from the more exact collision formulas given in [13].

Observe that electron-neutral collisions are dominant in the temperature elevation region, while the Coulomb collisions are large in the background region. These calculations agree with the observations that the cool electron population is observed only in the temperature elevation region.

\section{Plasma Source and Wave Launcher}

In the higher density regime the size of the temperature elevation depends upon the source tuning because of the resulting changes in plasma density. Changes in density result in changes in the wave power per particle and in the coupling between the wave launcher and wave. At a particular wave frequency a particular density is required near the wave launcher in order to couple effectively to the electromagnetic whistler mode (for $f_{p}>f$ ) with a parallel wave vector. If the coupling is not appropriate the wave will not be strong enough near cold electron-cyclotron resonance and the temperature elevation will be small.

The size of the temperature elevation also depends on the dc magnetic field, or equivalently the plasma-source axial position, because effective plasma production can occur only for a particular range of relative electron-cyclotron resonance positions in the cavity. These axial positions are typically in the range $-28<z<-25 \mathrm{~cm}$. This range for the $2.45-\mathrm{GHz}$ source resonance corresponds to that with the same magnetic field occurring at least $3 \mathrm{~cm}$ towards the wave launcher from the position
TABLE II

Calculated Collision Frequencies

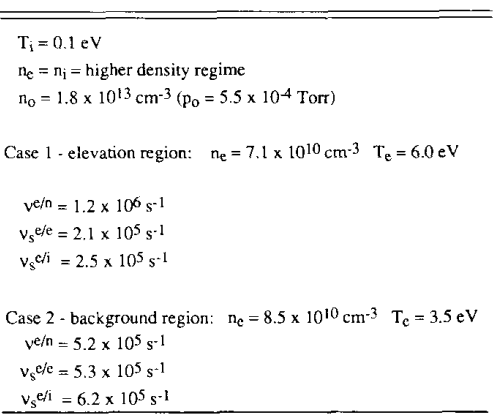

of the peak of the temperature elevation. For some ranges of magnetic field, fluctuations in the plasma density become large and accurate Langmuir probe traces cannot be obtained. The size of the temperature elevation is relatively insensitive to the axial position of the wave launcher. It is, however, for $f_{p}>f$ sensitive to the radial position of the wave launcher. The wave launcher must be symmetrically placed on-axis or the temperature elevation will be small or not present at all. This suggests that the excitation must be symmetrical to launch the proper wave for $f_{p}>f$.

\section{Mechanism for Temperature Elevation}

In addition to the launched wave propagation characteristics, we have seen that the probe itself plays an in tegral part in the formation of the temperature elevation. The two-probe correlation measurements indicate that the temperature elevation is localized $1-2-\mathrm{cm}$ radially about the probe tip and along a flux line. The power reflection coefficient from the wave launcher varies as a function of the probe position and probe type when $f_{p}>f$ by 10 percent. This is evidence for a substantial interaction between the launched wave and Langmuir probe. We also note that there was little correlation between the measured net helix coupled power variation and the temperature elevation axial profile as the probe was scanned through the cyclotron resonance zone. We believe that heating of electrons in a sheath resonance occurs near the probe tip caused by the interaction of the wave, the plasma sheath, and local probe boundary conditions at spacial positions somewhat below the Doppler-shifted cyclotron resonance. The wave power must be strong enough compared to the background plasma energy density near the probe tip to allow this heating.

We see a similar relative axial received wave profile structure down to very low launched power levels, but no temperature elevation until the power becomes sufficiently strong. This indicates that the probe response is not a function of the wave power but that the heating resulting from the resonance is. The input impedance of the probe tip and sheath plays a large role due to its influence on plasma wave-induced currents across the sheath which influence the electron heating and the fields around the 
probe tip. The proximity of the probe resonance to the cold electron-cyclotron resonance has an important influence on how effectively the electrons can absorb energy from the interaction. We have seen cases where a peaking of the received power from the coaxially shielded probe occurs far from the cold electron-cyclotron resonance position with no resulting temperature elevation. Two probe experiments indicate that the axial position of one probe relative to the other influences the locations of enhanced received power. This indicates that the experimental configuration with multiple probes can substantially influence the resulting wave fields. This work has shown that the observed wave probe-induced heating can be eliminated by the appropriate probe design by means of a high inductive RF impedance probe.

\section{ACKNOWLEDGMENT}

The authors wish to thank K.-O. Dai for computer programming, $\mathrm{K}$. Wiese for technical assistance, and $\mathrm{M}$. Bettenhausen for programming the numerical solution of electron-cyclotron wave damping in drifting bi-Maxwellian electron velocity distributions.

\section{REFERENCES}

[1] B. W. Rice and J. E. Scharer, IEEE Trans. Plasma Sci., vol. PS-14, p. 17, Feb. 1986

[2] B. D. McVey and J. E. Scharer, Phys. Rev. Lett., vol. 31, p. 1, 1973

[3] B. D. McVey and J. E. Scharer, Phys. Fluids, vol. 17, p. 142, 1974

[4] T. L. Owens and J. E. Scharer, Plasma Phys, vol 18, p. 663, 1976

[5] J. E. Scharer and J. E. Mitzlaff, Plasma Phys., vol. 19, p. 413, 1977

[6] J. H. Booske, W. D. Getty, R. M. Gilgenback, and R. A. Jong, Phys. Fluids, vol. 28 , p. 3116,1985

[7] J. R. Smith, N. Hershkowitz, and P. Coakley, Rev. Sci. Instrum. vol. 50, p. $210,1979$.

[8] G. Csanak, D. C. Cartwright, S. K. Srivastava, and S. Trajmar, in Electron-Molecule Interactions and Their Applications, vol. 1, L. G. Christophorous, Ed. New York: Academic, 1984, p. 2

[9] L. G. Christophorou, Atomic and Molecular Radiation Physics. New York: Wiley Interscience, 1971 , chaps. 4, 5, pp. 211-212, 379

[10] D. B. Batchelor, R. C. Goldfinger, and H. Weitzner, IEEE Trans. Plasma Sci., vol. PS-8, p. 78, June 1980
[11] T. H. Stix, The Theory of Plasma Waves. New York: McGrawHill, 1962, chaps. 9, 10.

[12] A. Fruchtman, K. Reidel, H. Weitzner, and D. B. Batchelor, Phys. Fluids, vol. 30, p. 115, 1987 .

[13] D. L. Book, NRL Plasma Formulary, Lab, for Computational Phys.. Naval Res. Lab., Washington, DC, 1987, 31 pp.

Richard R. Mett was born in Milwaukee, W1, in 1960. He received the B.S. degree in electrical engineering in 1982 from the Milwaukee Schoo of Engineering, and the M.S. degree in electrical engineering from the University of California, Berkeley, in 1985. He then became Chief Engineer at Dukar, Ltd. in Milwaukee. He reentered graduate school at the University of Wisconsin. Madison, in 1986 in the Department of Electrica and Computer Engineering, where he is currently a Research Assistant and $\mathrm{Ph}$. D . dissertator. His current interests include plasma waves and RF current drive.

Shu Wai Lam received the M.S. degree in physics from the University of Oregon, Eugene, in 1984. He is currently a Research Assistant in the Department of Electrical and Computer Engineering of the University of Wisconsin, Madison, where he is working towards the Ph.D. degree. His research interests are in plasma waves, plasma diagnostics, and plasma processing.

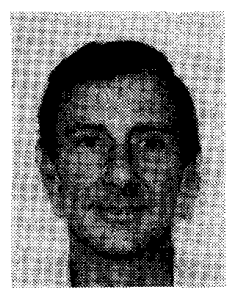

John E. Scharer was born in Monroe. WI, in 1939. He received the B.S., M.S., and Ph.D. de grees in electrical engineering from the University of California, Berkeley, in plasma physics.

$\mathrm{He}$ is a Professor with the Department of Electrical and Computer Engineering, University of Wisconsin, Madison. He has spent research sabbaticals at the CEA, Fontenay-aux-Roses, France. in 1970, and at the JET Tokamak in Culham, England, in 1983. He has been active with graduate students and scientists in theoretical, computational, and experimental research in the areas of linear and nonlinear plasma waves and the heating of fusion plasmas and lasers. He has also taught and recently started research in the area of free-electron lasers and coherent sources of radiation.

Dr. Scharer is a member of Tau Beta Pi and the American Physical Society. 